\title{
Petermann-factor limited sensing near an exceptional point
}

\author{
H. Wang, Y.-H. Lai, Z. Yuan, M.-G. Suh and K. Vahala* \\ ${ }^{1}$ T. J. Watson Laboratory of Applied Physics, California Institute of Technology, Pasadena, CA 91125, USA. \\ *vahala@caltech.edu
}

\begin{abstract}
We show that enhanced responsivity of a laser gyroscope operating near an exceptional point is accompanied by laser linewidth broadening related to the Petermann factor. The broadening imposes a fundamental sensitivity limit. (C) 2020 The Author(s)

OCIS codes: (140.3945) Microcavities; (190.0190) Nonlinear optics; (120.5790) Sagnac effect
\end{abstract}

Exceptional points (EPs) [1] have been experimentally realized [2] and applied to demonstrate improved opticalbased sensors [3,4]. The fundamental sensitivity limits in these EP-enhanced systems has attracted considerable interest. Recently, a strong scale factor improvement was reported in laser gyroscopes operating near an EP [5,6]. One report also observed increasing random-walk noise near the EP [5]. Here we show that this increasing noise is induced by mode non-orthogonality and that it compensates the responsivity enhancement. Thus, while operation of the gyroscope near the EP can potentially overcome technical sources of noise, the fundamental sensitivity limit (signal-to-noise ratio, SNR) does not improve. The noise enhancement is given by the Petermann factor [7]. While the connection of this factor to EPs has gained continued theoretical interest, the observation of Petermann linewidth broadening near EPs was reported only recently [8]. And the link between Petermann-factor-induced noise and EP sensor performance has not so far been considered.

The gyroscope uses a high-Q silica whispering gallery resonator in a ring-laser configuration (Fig. 1a). Optical pumping of clockwise (cw) and counter-clockwise (ccw) directions on the same whispering-gallery mode index creates stimulated Brillouin lasers (SBLs) [9]. Pump frequency detuning difference $\Delta \omega_{\mathrm{P}}$ is varied by acousto-optic modulation of a single laser. This, in turn, controls the cw and ccw SBL resonant frequencies as described in [5]. Dissipative backscattering couples the SBLs which creates an EP in the system. Rotation of the resonator perturbs SBL resonance frequencies, whose transduction into the SBL beating frequency $\Delta \omega_{\mathrm{S}}$ is enhanced relative to the conventional Sagnac scale factor by the signal enhancement factor SEF $=\left(\Delta \omega_{\mathrm{P}}-\Delta \omega_{0}\right)^{2} /\left[\left(\Delta \omega_{\mathrm{P}}-\Delta \omega_{0}\right)^{2}-\Delta \omega_{\mathrm{C}}^{2}\right]$ [5], where $\Delta \omega_{0}$ is a frequency offset related to the SBL Kerr effect and $\Delta \omega_{\mathrm{C}}$ is the critical value of $\left|\Delta \omega_{\mathrm{P}}-\Delta \omega_{0}\right|$ at which the system is biased at the EP.

As illustrated in Fig. 1b, the peculiar properties of non-orthogonal systems near the EP cause the composition of the optical modes to dramatically change near the EP. The fields experience a renormalization and fundamental noise is enhanced as illustrated in Fig. 1c. Because the laser linewidth can be understood to result from diffusion of the phasor (Fig. 1c), the SBL linewidth increases upon operation close to the EP. The linewidth enhancement is
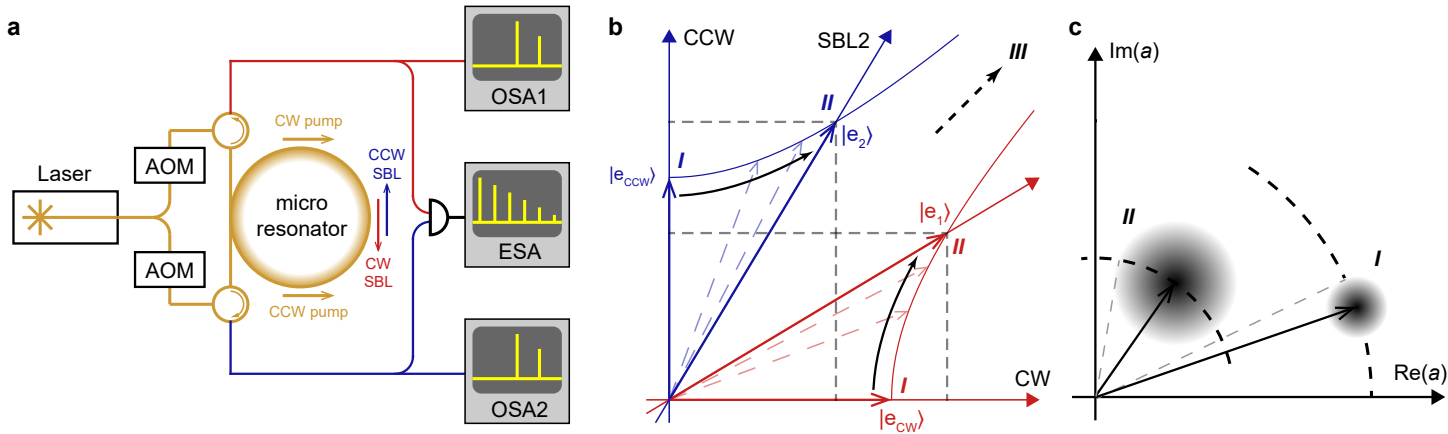

Fig. 1. (a), Experimental setup. AOM: acousto-optic modulator, OSA: optical spectrum analyzer, ESA: electrical spectrum analyzer. (b), Illustration of the cw-ccw and SBL1-SBL2 coordinate system at several bias points relative to the EP. Unit vectors for states $I$ and $I$ are shown on each axis. As the system is steered towards the EP, the SBL axes move toward each other so that unit vectors along the SBL axes lengthen. (c), The effective field phasor length is shortened and noise is enhanced as the system is steered to the EP. 

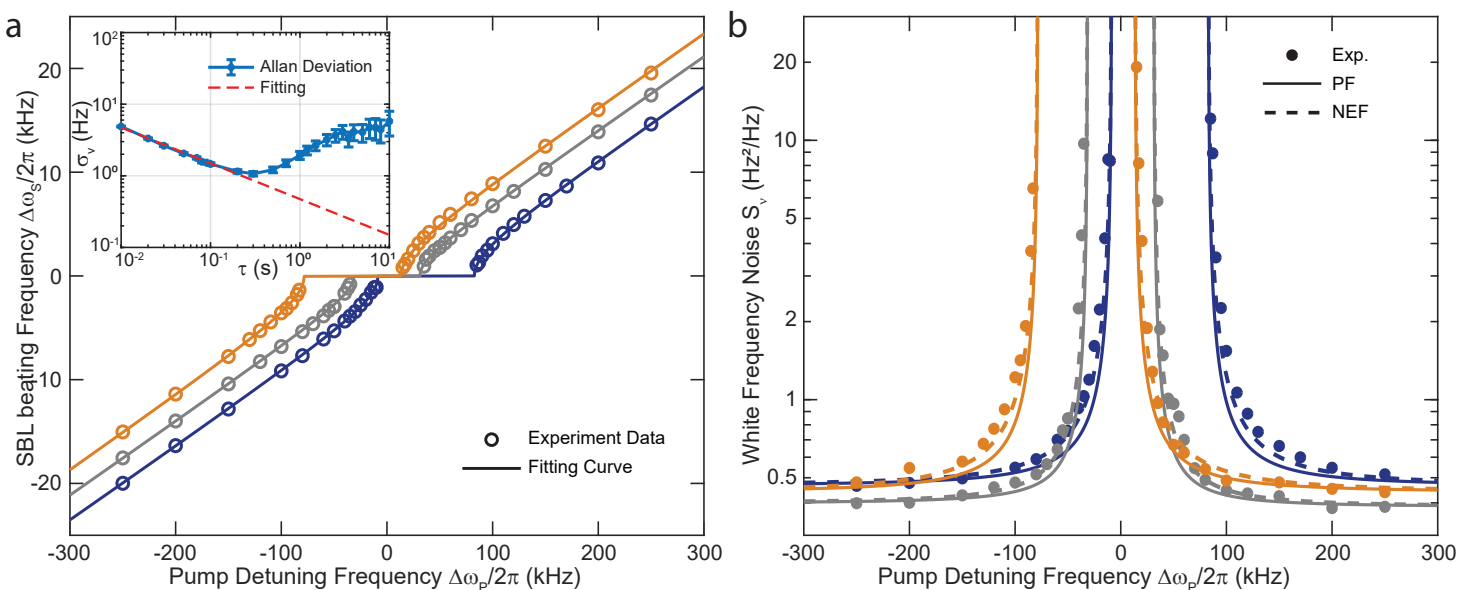

Fig. 2. (a), Measured SBL beating frequency is plotted versus pump detuning. Data point color corresponds to different $\Delta \omega_{0}$ values. Solid curves are theoretical fittings. Inset is a typical Allan deviation of frequency $\sigma_{v}(\tau)$ versus gate time $\tau$. The short-term part is fitted with $\sqrt{S_{v} /(2 \tau)}$ where $S_{V}$ is the one-sided power spectral density of the white frequency noise plotted in panel b. (b), Measured white frequency noise level of the beating signal. Data point color corresponds to panel a. Petermann factor PF (solid lines) and the NEF (dashed lines) theoretical predictions use parameters obtained by fitting from panel $\mathbf{a}$.

given by the Petermann factor, which, for this system, can be shown to be given by PF $=\left(\Delta \omega_{\mathrm{P}}-\Delta \omega_{0}\right)^{2} /\left[\left(\Delta \omega_{\mathrm{P}}-\right.\right.$ $\left.\left.\Delta \omega_{0}\right)^{2}-\Delta \omega_{\mathrm{C}}^{2}\right]$. Inspection shows that PF $=\mathrm{SEF}$, and as a result the SNR is not expected to improve near the EP when the system is fundamental-noise limited.

To verify the above predictions, $\Delta \omega_{P}$ is varied using the acousto-optic modulators. This permits precise variation of both the SEF and the PF. To measure noise, the SBL beating frequency $\Delta \omega_{S}$ is measured by a frequency counter (Fig. 2a) and analyzed in an Allan deviation (Adev) measurement (Fig. 2a inset). $\Delta \omega_{0}$ was also varied to produce three data sets for plotting. The initial roll-off of the Adev features a slope of $-1 / 2$ corresponding to white frequency noise. The slope of this region is fit to $\sqrt{S_{V} /(2 \tau)}$ where $S_{v}$ is the one-sided spectral density of the white frequency noise. The inferred white frequency noise of the SBL beating signal at each detuning point is plotted in Fig. 2b. A frequency noise enhancement is observed as the system is biased towards the EP. Aside from a slight discrepancy, there is overall excellent agreement between measurement and the Petermann factor prediction. The frequency noise levels measured in Fig. $2 b$ are consistent with Petermann-enhancement of a Schawlow-Townes linewidth (i.e., fundamental noise). To test the Petermann prediction, we also derived an Adler-like coupled-mode equation analysis for this system. It gives a noise enhancement factor NEF $=\left[\left(\Delta \omega_{\mathrm{P}}-\Delta \omega_{0}\right)^{2}+\Delta \omega_{\mathrm{C}}^{2} / 2\right] /\left[\left(\Delta \omega_{\mathrm{P}}-\right.\right.$ $\left.\left.\Delta \omega_{0}\right)^{2}-\Delta \omega_{\mathrm{C}}^{2}\right]$ that is close in value to the PF. The PF and NEF predictions are shown on Fig. $2 \mathrm{~b}$ and the NEF correction provides a slightly better agreement with the data at the intermediate detuning values.

In summary, we have verified through measurement and theory that mode non-orthogonality sets a fundamental limitation to a class of sensors operating near an EP. Remarkably, a resulting noise enhancement precisely compensates the sensor's EP-enhanced response. It is nonetheless important to note that when SNR is limited by technical noise considerations, it still could be advantageous to operate near the EP. It is also possible that other sensing modalities could benefit from operation near an EP.

This work was supported by DARPA (N66001-16-1-4046) and the Kavli Nanoscience Institute.

\section{References}

1. Bender, C. M. \& Boettcher, S. Physical Review Letters 80, 5243 (1998).

2. Feng, L., El-Ganainy, R. \& Ge, L. Nature Photonics 11, 752 (2017).

3. Wiersig, J. Physical Review Letters 112, 203901 (2014).

4. Chen, W., Özdemir, Ş. K., Zhao, G., Wiersig, J. \& Yang, L. Nature 548, 192 (2017).

5. Lai, Y., Lu, Y., Suh, M. et al. Nature 576, 65-69 (2019).

6. Hokmabadi, M.P., Schumer, A., Christodoulides, D.N et al. Nature 576, 70-74 (2019).

7. Petermann, K. IEEE Journal of Quantum Electronics 15, 566-570 (1979).

8. Zhang, J. Peng, B. Özdemir, Ş. K. et al. Nature Photonics 12, 479 (2018).

9. Li, J., Lee, H., Chen, T. \& Vahala, K. J. Optics Express 20, 20170-20180 (2012). 